

\title{
Efecto del Espesor de la Chapa sobre la Microestructura del Depósito de Recargue mediante Proceso SMAW
}

\author{
Tamara M. Ortiz Méndez ${ }^{\circledR}$, Amado Cruz-Crespo $@$, Juan A. Pozo Morejón
}

\author{
Centro de Investigaciones de Soldadura, Facultad de Ingeniería Mecánica e Industrial, \\ Universidad Central "Marta Abreu" de Las Villas, CP 54 830, Santa Clara, VC, Cuba. \\ *Autor de correspondencia: tortiz@uclv.cu \\ https://doi.org/10.22209/rt.v44n3a04
}

Recepción: 29 de septiembre de 2020 | Aceptación: 05 de junio de 2021 | Publicación: 01 de agosto 2021

\begin{abstract}
Resumen
El establecimiento de un procedimiento de recargue por soldadura pasa comúnmente por la evaluación previa del consumible a emplear mediante ensayos de caracterización del depósito, cuyos resultados deben ser reproducibles en la práctica. Las dimensiones de las planchas para la realización de los depósitos de ensayo determinan, junto al calor aportado, el ciclo térmico al que se somete el material del depósito; sin embargo, este aspecto frecuentemente no se toma en consideración al elaborar las muestras de ensayo. En el presente trabajo se estudió del efecto del espesor del metal base sobre el coeficiente de dilución, la microestructura y la dureza de depósitos de recargue constituidos por fundiciones blancas, basado en el empleo de los esquemas teóricos ideales de cuerpos y fuentes de calor, así como de modelos matemáticos aplicados en la teoría de los procesos térmicos en la soldadura. Se demostró el efecto significativo del parámetro espesor del metal base en las variables respuesta estudiadas y se comprobó además la aplicabilidad de modelos teóricos de los procesos térmicos en la soldadura en el adecuado dimensionamiento de las planchas para la realización de los depósitos de ensayo, lo que posibilita la reproducibilidad en la práctica de los resultados de la caracterización de materiales de recargue.
\end{abstract}

Palabras clave: fundición blanca aleada; procesos térmicos en la soldadura; recargue; soldadura.

\section{Effect of Plate's Thickness on the Hardfacing Welds Microstructure using SMAW Process}

\begin{abstract}
The establishment of hardfacing procedure by welding commonly goes through a previous evaluation of the consumable to be used, by means of the characterization tests of the welds; those results must be reproducible under practical conditions. The plate's sizes for hardfacing welds depositions determine, together with heat input, the thermal cycle undergone by the weld material; however this aspect is frequently avoided when the samples are elaborated. In the present work was studied the effect of parent metal thickness on the dilution coefficient, the microstructure and the hardness of the hardfacing welds formed by white irons, based on the bodies and heat sources simplified schemes, as well as the mathematical models applied in thermal processes in welding. The results demonstrated the significant effect of plate thickness parameter on the response variables studied and the applicability of theoretical models of thermal processes in welding is also demonstrated in the adequate sizing of the test plates, which allows the reproducibility in practice of the results of the characterization of hardfacing welds.
\end{abstract}

Keywords: hardfacing; thermic process in welding, white cast iron; welding.

\section{Introducción}

El recargue por soldadura es una de las técnicas más eficientes para la protección de elementos de máquinas contra diferentes condiciones de desgaste. El éxito de los procedimientos de recargue depende de la adecuada selección de diferentes variables vinculadas al tipo de metal de aporte, al proceso de soldadura empleado, a los parámetros del régimen (calor aportado) y a las condiciones de disipación de calor (Gucwa et al., 2020). Para la elaboración de estos procedimientos generalmente se realizan ensayos previos con diferentes materiales de aporte y regímenes, en función de estudiar su efecto en las propiedades del depósito, lo que permite 
obtener la combinación más adecuada de estas variables y de las técnicas para su aplicación. La mayoría de las investigaciones reportadas relacionadas con el recargue de piezas, entre ellas las de Morsy y El-Kashif (2014), y Yüksel y Sahin (2014), están dirigidas a desarrollar una solución para enfrentar el desgaste abrasivo, debido a su prevalencia en ramas como la minería, la agricultura, la construcción, la industria azucarera y el transporte, con una incidencia entre el 50 y $60 \%$ del volumen total de piezas desgastadas. Dichas piezas generalmente son voluminosas, con gran diversidad de formas y dimensiones (ej. surcadores, cuchillas picadoras y desmenuzadoras, dientes de zanjadoras, etc.) (Dulón-Gómez, 2003). Ello impone determinado nivel de severidad en las condiciones de disipación de calor desde el depósito, que generalmente no es considerado en el dimensionamiento de las chapas al realizar depósitos de recargue para la evaluación de los consumibles a emplear.

Los ciclos térmicos generados en la zona del depósito varían, para igual régimen de soldeo, en dependencia del espesor y de la geometría del material base, de la longitud del depósito y de la posición de la zona de ensayo, así como de las condiciones de intercambio con el medio, estableciendo determinadas condiciones de disipación del calor, que condicionan el proceso de cristalización desde la fase líquida y la microestructura final. Dichas condiciones manifiestan su efecto en el gradiente térmico, $(\mathrm{G})$, la razón de solidificación en la interfase (R), y la velocidad de enfriamiento en la interfase a la temperatura de fusión (GR), determinando la microestructura y las propiedades del material del depósito (Masubuchi, 1980; Olson, 1993). Esto ha sido demostrado en un estudio donde se reguló el tamaño de carburos en depósitos de recargue, constituidos por fundiciones blancas de alto cromo, mediante el control de la disipación de calor (Gucwa et al., 2020). En este caso se obtuvieron diferencias significativas en la estructura de los depósitos, concernientes a la forma y tamaño de los carburos. Las microestructuras hipereutécticas de mayor fracción volumétrica de carburos primarios y dureza, se formaron en depósitos enfriados en condiciones de mayor razón de disipación de calor, lo que se reflejó en la resistencia al desgaste erosivo.

Como es evidente, las condiciones de disipación también tienen un efecto en la geometría del depósito y por tanto en la dilución, al influenciar directamente en la forma y dimensiones de las isotermas de los campos térmicos.

A pesar de lo anterior, sorprende el hecho de que la forma y dimensiones del metal base donde se deposita el recargue para su caracterización química, metalográfica, de dilución, de dureza, de resistencia al desgaste, entre otras, no se aborde con frecuencia como una variable de entrada para la experimentación. Tampoco se observa uniformidad de criterios a la hora del dimensionamiento de la muestra, de manera que se simulen los ciclos térmicos que se obtendrían al depositar sobre la pieza a la que se destina el estudio. Esto tiene un efecto en la eficacia del procedimiento posterior de recargue, ya que se afecta la reproducibilidad de los resultados del ensayo en la aplicación práctica sobre la pieza a recargar.

Con vistas a establecer criterios para el dimensionamiento de las muestras de ensayo empleadas en la caracterización de depósitos de recargues, el presente trabajo se trazó como objetivo el estudio del efecto del espesor del metal base sobre el coeficiente de dilución, la microestructura y la dureza de depósitos constituidos por fundiciones blancas, basado en el empleo de los esquemas teóricos ideales de cuerpos y fuentes de calor, así como de modelos matemáticos aplicados en la teoría de los procesos térmicos en la soldadura, para fundamentar el fenómeno de transferencia de calor desde el depósito de recargue.

\section{Materiales y Métodos}

Para la obtención de los depósitos fue empleado un electrodo tubular revestido CIS(0,1-0,25), de 4 mm de diámetro, desarrollado en el Centro de Investigaciones de Soldadura, de la Universidad Central "Marta Abreu" de Las Villas (Ortiz-Méndez, 2018). Este nuevo material fue estudiado desde el punto de vista de su comportamiento operacional y de las características del depósito sin dilución, por lo cual se conoce que el material está constituido por una fundición blanca hipereutéctica, aleada con Cr, Mn y Si (Tabla 1), cuyos contenidos se enmarcan en las composiciones recomendadas por la norma AWS A5.13 (AWS, 2010), para el recargue de piezas sometidas a desgaste abrasivo como mecanismo principal de deterioro.

En el presente estudio, la variable de entrada de los experimentos fue el espesor de la chapa empleada como material base, manteniendo constante el calor aportado, con lo cual se establecen diferentes condiciones de disipación del calor desde el depósito ensayado. Para ello, se empleó un régimen de soldadura único, con una corriente $(I)$ de $120 \mathrm{~A}$, valor con el cual opera satisfactoriamente el electrodo CIS $(0,1-0,25)$; una tensión de arco $(U)$ de $27 \mathrm{~V}$ y una velocidad media de soldadura $\left(v_{s}\right)$, medida experimentalmente, de 10,0 $\pm 0,7 \mathrm{~m} / \mathrm{h}$ (Ortiz-Méndez, 2018). Con estos parámetros fue determinado el calor de entrada o heat input $\left(H_{L}\right)$, mediante la Ecuación 1 , 
tomando una eficiencia del proceso $(\eta)$ de $80 \%$ (Olson et al., 1993), con lo cual se obtuvo un valor de 9,35.10 $\mathrm{J} / \mathrm{m}$.

$$
H_{L}=\eta\left(\frac{I \cdot U}{v_{s}}\right)
$$

Las variables respuestas fueron la dilución, la morfología de la microestructura y la dureza media del depósito de recargue. Esta última es un indicador de las propiedades de resistencia al desgaste cuando se trata de comparar depósitos de composiciones químicas similares, dentro de un mismo sistema de aleación (Chotěborský et al., 2008). Los depósitos de ensayo, de cordón simple, una pasada y sin oscilación, se llevaron a cabo mediante el proceso de soldadura manual con electrodo revestido (SMAW, según sus siglas en inglés), con una fuente tipo inversor. Estos fueron realizados sobre chapas de acero AISI 1020 (Tabla 1).

Tabla 1. Composición química del metal base y metal de aporte sin dilución (\% en masa).

\begin{tabular}{lccccccc}
\hline \multicolumn{1}{c}{ Material } & C & Cr & Mn & Si & P & S & Fe (balance) \\
\hline Metal de aporte CIS(0,1-0,25) & 3,27 & 12,34 & 4,09 & 2,78 & 0,018 & 0,009 & 77,50 \\
Metal base (AISI 1020) & 0,26 & 0 & 1,00 & 0,40 & 0,040 & 0,050 & 98,25 \\
\hline
\end{tabular}

Para la determinación de la geometría y la observación metalográfica de los depósitos obtenidos, se extrajeron muestras mediante cortes transversales en una tronzadora metalográfica, con el empleo de líquido refrigerante para evitar el calentamiento de la muestra. La preparación de las muestras se realizó de acuerdo con la norma ASTM E 3 (ASTM, 2011), mediante desbaste y pulido, con suspensión de óxido de cromo de $3 \mu \mathrm{m}$; el ataque fue realizado con Nital al $2 \%$. Las muestras fueron observadas en un microscópico óptico metalográfico acoplado a una computadora para la adquisición de imágenes.

Con ayuda del programa SigmaScanPro se midieron las áreas de penetración $(A p)$ y del refuerzo $(A r)$ en la sección transversal de los depósitos, y con ello calculado el coeficiente de dilución $(D)$, mediante la Ecuación 2 (Oates et al., 1998).

$$
D(\%)=\frac{A p}{A p+A r} \cdot 100
$$

La dureza de la zona central del cordón fue determinada con un microdurómetro Vicker, con carga de $1000 \mathrm{~g}$ y 12 min de tiempo de indentación. Se realizaron 3 mediciones en cada caso, con lo que fue determinado el valor medio. Para estimar las fracciones de volumen de los constituyentes microestructurales, se empleó la técnica de conteo de puntos establecida por la norma ASTM E562 (ASTM, 2011).

\section{Determinación de los espesores de chapas para la realización de los depósitos}

Con el objetivo de determinar un rango de espesores de las muestras de ensayo que abarcara las condiciones extremas de transferencia del calor (disipación mínima y máxima a partir del depósito), para el régimen de soldadura dado, se emplearon los "esquemas de cálculo simplificado de cuerpos y de fuente de calor" (Grong, 1997; Marques y Modenesi, 2014). Estos modelos son soluciones analíticas de ecuaciones diferenciales, sujetos a simplificaciones, por lo que el grado de representatividad de la realidad dependerá de la elección correcta de dichos esquemas. Para su empleo se considera que toda la disipación de calor desde el depósito se realiza por conducción, debido a que este es el mecanismo más severo cuando se trata de sólidos rodeados por aire sin corriente forzada.

Según los autores antes referidos, el modelo de chapa gruesa o cuerpo seminfinito representa un tipo de cuerpo cuyas dimensiones son tales que todas las superficies límites, excepto el plano sobre el cual se efectúa el depósito, están lo suficientemente alejadas de la fuente de calor por lo que su presencia no modifica la distribución de las isotermas y los ciclos térmicos en la zona cercana al cordón, transfiriéndose este de manera tridimensional desde las zonas más calientes hacia las más frías. Su empleo se recomienda para analizar el proceso de transferencia de calor durante el depósito de un cordón sobre una chapa de dimensiones significativas, lo que ilustra las condiciones del recargue sobre piezas de grandes dimensiones. En este modelo se considera, además, una fuente de calor puntual con desplazamiento constante. El espesor de la chapa crítico o límite $\left(\mathrm{d}_{\mathrm{c}}\right)$ a partir del cual se aplica el modelo de cuerpo seminfinito se calcula mediante la Ecuación 3 (Marques y Modenesi, 2014). A partir de este espesor se considera que ocurre la condición de máxima disipación del calor desde el depósito, para un régimen dado de soldadura. Se ha demostrado que estos modelos teóricos garantizan la mayor precisión cuando se emplean valores medios de las propiedades termodependientes, entre las correspondientes a la temperatura ambiente y la del sólido de la aleación (Pozo et al., 2018). 


$$
(d c)_{T 2 / T 1}=\sqrt{\frac{H_{L}}{2 \rho C}\left[\frac{1}{T_{2}-T_{0}}+\frac{1}{T_{1}-T_{0}}\right]}
$$

Donde: $\rho$ es la densidad del acero $\left(7530 \mathrm{~kg} / \mathrm{m}^{3}\right), c$ es el calor específico $(750 \mathrm{~J} / \mathrm{kg} \cdot \mathrm{K})$, ambos valores medios entre la temperatura ambiente y $\left.1500{ }^{\circ} \mathrm{C}\right)(\mathrm{EN}, 2005) ; H_{L}$ es la energía lineal de soldadura $\left(9,35 \cdot 10^{5} \mathrm{~J} / \mathrm{m}\right) \mathrm{calculada}, T_{0}$ es la temperatura ambiente (se asume $25^{\circ} \mathrm{C}$, despreciando el error de no considerar el efecto previo del arco). $T_{1} y$ $T_{2}$ sin los valores máximo y mínimo del rango de temperatura de interés.

Para la definición de estos valores se tuvo en consideración que durante la cristalización, el material del depósito pasa por diferentes rangos de temperaturas críticas, los cuales tienen un efecto en la estructura metalográfica y las propiedades del material (Guliaev, 1978). Basado en lo anterior, es necesario conocer la composición química del material del depósito, lo que permite definir el rango de temperatura de interés. Para ello, se calculó la composición química media del depósito, empleando un coeficiente de dilución de 0,4 ; obtenido en estudios previos con el metal de aporte CIS(0,1-0,25) y el régimen empleado (Ortiz-Méndez, 2018). La composición obtenida fue de $2 \%$ de $\mathrm{C} ; 6,57 \%$ de $\mathrm{Cr} ; 2,6 \%$ de $\mathrm{Mn}$ y $1,7 \%$ de $\mathrm{Si}$, con contenidos de $\mathrm{P}$ y $\mathrm{S}$ de 0,018 y $0,009 \%$, respectivamente. Según el diagrama de proyección del liquidus del sistema ternario $\mathrm{Fe}-\mathrm{Cr}-\mathrm{C}$ con $6 \%$ de cromo (Thum, 1935; Schön, y Sinatora, 1998), esta composición química se corresponde con una fundición blanca aleada hipoeutéctica, con una microestructura constituida de componente eutéctico, en la región interdendrítica de austenita primaria.

Ha sido demostrado el efecto de la fracción de volumen de la fase de formación primaria en las características de dureza y resistencia al desgaste de depósitos de recargue compuestos por fundiciones blancas, con dependencias directamente proporcionales entre ambas, lo cual se explica dado el efecto de los carburos eutécticos como barreras a la deformación del material (Chotěborský et al., 2011; Ortiz-Méndez et al., 2019). Basado en esto, el rango de temperaturas en que ocurre la nucleación y crecimiento de esta fase es de especial importancia en este análisis y se toma como base para la determinación del espesor crítico. Al consultar la sección transversal del diagrama $\mathrm{Fe}-\mathrm{Cr}-\mathrm{C}$ con $6 \%$ de $\mathrm{Cr}$, se observó que el rango aproximado de temperaturas donde ocurre la formación y crecimiento de la estructura de cristalización primaria, para la composición química estimada, se encuentra entre 1150 y $1430^{\circ} \mathrm{C}$ (Thum, 1935; Schön, y Sinatora, 1998), por tanto, el tiempo de permanencia en él determina la fracción volumétrica de las fases del depósito. A partir de lo anterior se establece, para la determinación del espesor crítico, un rango más amplio de temperaturas, 1100 y $1500{ }^{\circ} \mathrm{C}$, considerando el efecto del subenfriamiento en las temperaturas críticas y la influencia de los elementos de aleación en las regiones de existencia de las fases (Guliaev, 1978). Sustituyendo estos valores en la Ecuación 3, se obtuvo un espesor crítico de $14 \mathrm{~mm}$, con lo cual se establece la condición de máxima disipación de calor desde el depósito, que en teoría se mantiene para un espesor superior a este.

El esquema ideal de chapa fina ilustra el caso del espesor de chapa para la condición límite de mínima disipación, el cual representa un cuerpo limitado por dos planos paralelos $\mathrm{z}=0 \mathrm{y} \mathrm{z}=\mathrm{d}$, donde se considera que la temperatura a lo largo de todo el espesor es uniforme y que el calor solo se transfiere en direcciones paralelas a la superficie (transferencia bidimensional) (Grong, 1997). Se recomienda el empleo de este modelo en soldaduras de chapa con una sola pasada. En el recargue (cordón sobre chapa) estas condiciones se manifiestan cuando ocurre el sobrecalentamiento o la perforación de la chapa, lo cual ocurrió en la práctica, para las condiciones de soldadura empleadas en chapas de espesores menores a $4 \mathrm{~mm}$, por lo cual este fue seleccionado como espesor mínimo para los ensayos, donde se establecen las menores tasas de disipación de calor desde el depósito.

Otra consideración a tener en cuenta para el empleo de los modelos teóricos de análisis de transferencia de calor en la soldadura, es que esta ocurre en estado cuasiestacionario desde la zona calentada por la fuente y, por tanto, la distribución de temperatura resulta estacionaria referida a un sistema de coordenadas móviles que se desplaza junto a la fuente de calor (Masubuchi, 1980; Grong, 1997). Dicho estado se logra a determinada distancia desde el inicio del cordón de soldadura, luego de estabilizarse la temperatura alrededor de la fuente de calor (termosaturación), y antes de que ocurra la etapa de nivelación, después de concluido el depósito. Para garantizar lo anterior se realizaron cordones de longitud $200 \mathrm{~mm}$ y las muestras de ensayo del depósito se extrajeron a una distancia de $100 \mathrm{~mm}$ después del inicio del cordón. La longitud de las chapas fue de $300 \mathrm{~mm}$ de largo y $100 \mathrm{~mm}$ de ancho, lo cual garantizó una distancia de $100 \mathrm{~mm}$ a cada lado del depósito y $50 \mathrm{~mm}$ previo y posterior al cordón, con vistas a separarlo suficientemente de los bordes de la chapa, de manera que la influencia en la transferencia de calor de las condiciones de frontera en esa dirección sea despreciable.

Basado en el análisis anterior, se establecieron 4 puntos experimentales. Además de los espesores de 4 y $14 \mathrm{~mm}$, con los que se establecieron las condiciones mínima y máxima de disipación del calor, se incluyeron otros dos de 8 y de $16 \mathrm{~mm}$, con el objetivo de lograr la descripción precisa del efecto del espesor de la chapa de metal base en las propiedades del depósito (Tabla 2). Se realizaron tres réplicas para cada condición de ensayo, por lo 
cual las letras que identifican la denominación de las muestras fueron acompañadas por números que identifican la réplica.

Tabla 2. Clasificación de las muestras de ensayo para el estudio de microestructura y dureza.

\begin{tabular}{cc}
\hline Muestras & Espesor (mm) \\
\hline A $(1,2,3)$ & 4 \\
B $(1,2,3)$ & 8 \\
C $(1,2,3)$ & 14 \\
D $(1,2,3)$ & 16 \\
\hline
\end{tabular}

\section{Resultados y Discusión}

\section{Análisis de los resultados de dilución de los depósitos}

En la Tabla 3 se exponen los coeficientes de dilución medios, calculados a partir de la medición experimental de las áreas de los depósitos. Puede apreciarse que la dilución disminuyó con el incremento del espesor de la chapa, para los espesores desde 4 hasta $14 \mathrm{~mm}$. Esto evidencia un efecto en los campos térmicos de estrechamiento de las isotermas a medida que aumenta el espesor, dado que se facilita la transferencia de calor por conducción desde la zona más caliente del cordón hasta la más fría, alejada de este. Los resultados de dilución en los depósitos sobre las chapas de 14 y $16 \mathrm{~mm}$ fueron semejantes. Esto es señal de que a partir de los $14 \mathrm{~mm}$ de espesor de metal base, la superficie límite inferior de la chapa deja de ejercer un efecto de frontera de la conducción de calor y su presencia no modifica los campos térmicos generados por el efecto de la fuente de calor, para el régimen de soldadura y el metal base utilizados, lo cual apoya la selección del modelo empleado para la determinación del espesor crítico.

Con el empleo de los coeficientes de dilución obtenidos experimentalmente (D) y los datos de composición del metal de aporte (\%Exma) y el metal base (\%Exmb), se estimó el contenido medio de los diferentes elementos de aleación en el depósito (\%Ex) mediante la Ecuación 4 (Oates, 1998) (Tabla 3). Los resultados demuestran el efecto de la velocidad de enfriamiento del depósito desde la fase líquida en su composición química.

$$
\% E_{x}=\left(D * \% E_{X M B}\right)+\left[(1-D) * \% E_{X M A}\right]
$$

Tabla 3. Coeficientes de dilución medio y composición química estimada en los depósitos de recargue.

\begin{tabular}{ccccccccc}
\hline Muestra & $\begin{array}{c}\text { Espesor } \\
(\mathbf{m m})\end{array}$ & $\begin{array}{c}\text { Coeficiente de dilución } \\
\text { medio, D }(\boldsymbol{\%})\end{array}$ & \multicolumn{5}{c}{ Composición estimada de los depósitos de ensayo } \\
& & $\mathbf{C}$ & $\mathbf{C r}$ & $\mathbf{M n}$ & $\mathbf{S i}$ & $\mathbf{P}$ & $\mathbf{S}$ \\
\hline $\mathrm{A}$ & 4 & 0,480 & 1,83 & 6,42 & 2,61 & 1,64 & 0,03 & 0,03 \\
$\mathrm{~B}$ & 8 & 0,443 & 1,95 & 6,91 & 2,73 & 1,73 & 0,03 & 0,03 \\
$\mathrm{C}$ & 14 & 0,381 & 2,13 & 7,65 & 2,92 & 1,88 & 0,03 & 0,02 \\
$\mathrm{D}$ & 16 & 0,377 & 2,16 & 7,77 & 2,95 & 1,90 & 0,03 & 0,02 \\
\hline
\end{tabular}

En todos los casos, el material de los depósitos analizados se enmarca en el sistema de las fundiciones blancas aleadas hipoeutécticas (Raynor y Rivlin, 1988). La variación del espesor del metal base, desde las condiciones máximas a las mínimas de disipación de calor, tiene un efecto en la composición química del depósito en el orden de las 3 décimas porcentuales para el carbono, el manganeso y el silicio, y superior a la unidad para el cromo, lo que influye marcadamente en su microestructura y propiedades finales, considerando la complejidad y sensibilidad térmica de los procesos por los que transitan las fundiciones blancas hipoeutécticas durante su enfriamiento.

\section{Análisis de los resultados de microestructura de los depósitos}

En la Figura 1 se muestran las microestructuras obtenidas en la zona central de la sección transversal de los depósitos analizados. Considerando que los depósitos fueron realizados con igual material de aporte y régimen de soldadura, este resultado ilustra el efecto de la variación de las condiciones de disipación del calor en la estructura metalográfica del depósito, dado su efecto en el ciclo térmico al que se somete el material. 


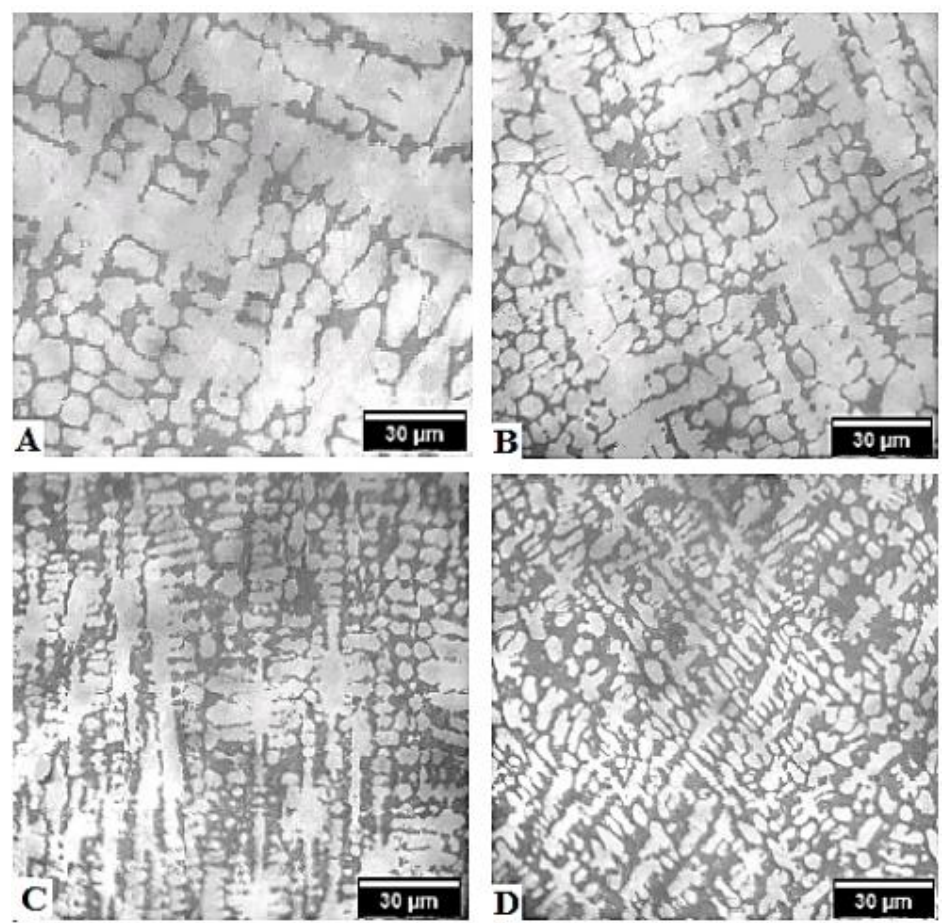

Figura 1. Microestructuras típicas obtenidas en los depósitos de ensayo con diferentes espesores de chapa, A-4 $\mathrm{mm}, \mathrm{B}-8 \mathrm{~mm}, \mathrm{C}-14 \mathrm{~mm}, \mathrm{D}-16 \mathrm{~mm}$.

En todas las imágenes se observan dos zonas de coloración diferente (clara y oscura), que varían en forma y tamaño para las diferentes condiciones de ensayo. Esta microestructura tiene una apariencia típica de una fundición blanca hipoeutéctica, con morfologías resultantes de los modos de solidificación columnar y equiaxial dentrítico, típicas de la cristalización a elevados grados de subenfriamiento, propia de los depósitos de recargue (Kou, 2002; Collazo y López, 2009; Chotěborský et al., 2011).

Basado en la composición química (Tabla 3) y la morfología de las fases que se aprecian en las imágenes (Figura 1), se puede establecer que los constituyentes de la microestructura se corresponden con austenita primaria (zona clara) y mezcla eutéctica (zona oscura), compuesta por carburos aleados y austenita eutéctica (Raynor y Rivlin, 1988). Es común en este tipo de aleaciones que la austenita se mantenga a temperatura ambiente como una fase metastable, atribuible al contenido de elementos de aleación, fundamentalmente el Mn, como estabilizador de dicha fase (Matsuo et al., 2005). Se añade como causa de lo anterior, su alto contenido de aleación de no equilibrio, lo cual se debe a la redistribución del soluto durante la solidificación y a la baja cinética de precipitación de carburos secundarios, dado lo limitado de los procesos difusivos en estado sólido, bajo las condiciones de enfriamiento establecidas para los depósitos de recargue estudiados (Dieter, 1997; Vedia y Svoboda, 2004).

En la Figura 1 también se observan claramente las diferencias de áreas cubiertas por las dentritas de fase de cristalización primaria (zona clara) en los diferentes depósitos de ensayo. Las dentritas se afinan a medida que aumenta el espesor de la chapa como consecuencia de la formación de mayor número de centros de cristalización, debido al aumento del grado de subenfriamiento del metal del depósito en estado líquido, dado por la mayor velocidad de enfriamiento y a la disminución del volumen de metal fundido (menor dilución). Esto trae como resultado la formación de una estructura más fina en las condiciones de mayor disipación del calor (Kou, 2002). El área ocupada por la matriz metálica (zona clara) disminuye desde un valor medio de $85 \%$ en la zona central del depósito sobre una chapa de $4 \mathrm{~mm}$ de espesor, hasta $65 \%$ en la chapa de $16 \mathrm{~mm}$.

Las áreas correspondientes a la matriz austenitica en los depósitos con chapa de 14 y 16 mm fueron similares, con valores medios de 67 y $65 \%$, respectivamente. Esto corrobora nuevamente el empleo del espesor crítico como dimensión a partir de la cual no se modifican los campos y ciclos térmicos, y se demuestra la conveniencia del empleo de los esquemas ideales de cálculo, utilizados en la teorías clásica de los procesos térmicos de soldadura, en el estudio del efecto de las condiciones de disipación de calor sobre las características de la microestructura del depósito. 


\section{Análisis de los resultados de dureza de los depósitos}

En la Figura 2 se encuentran graficados los resultados de dureza media en la zona central de los depósitos, realizados en las muestras con diferente espesor de chapa. En todas las muestras se obtuvieron valores de dureza mayores a $400 \mathrm{HV}$, lo cual es típico de las fundiciones blancas aleadas, donde la matriz luego del enfriamiento, está constituida por estructuras de no equilibrio (Radzikowska, 2004). Se observa que la dureza crece marcadamente a medida que incrementa el espesor de la muestra de ensayo, obteniéndose una variación cercana a los $100 \mathrm{HV}$ entre los depósitos donde se establecen las condiciones de máxima y mínima disipación de calor (A y C). Este comportamiento es coherente con los resultados del análisis metalográfico de los depósitos. Las mayores durezas se obtuvieron en los depósitos de las muestras C (14 mm) y D $(16 \mathrm{~mm})$, lo cual coincide con resultados de otras investigaciones (Tabrett et al., 1996; Chotěborský et al., 2011), que demuestran que a medida que se afinan las dentritas de austenita primaria y aumenta la fracción volumétrica de mezcla eutéctica, en un depósito compuesto por una fundición blanca aleada hipoeutéctica, aumenta la dureza del material.

Otro aspecto a considerar, relativo al proceso de cristalización primaria, se relaciona con sus características en condiciones de enfriamiento continuo, típicas de los depósitos de recargue. Los primeros cristales de austenita primaria tienen por lo general una concentración más alta del componte menos fusible que los que se forman después. Los espacios interdentríticos que cristalizan de último, contienen la mayor cantidad de componentes más fusibles, debido a lo limitado de la difusión en estas condiciones de enfriamiento. Este fenómeno es conocido como segregación dentrítica y ocurrirá en diferente grado, gobernado por la velocidad de enfriamiento del depósito (Guliaev, 1978).

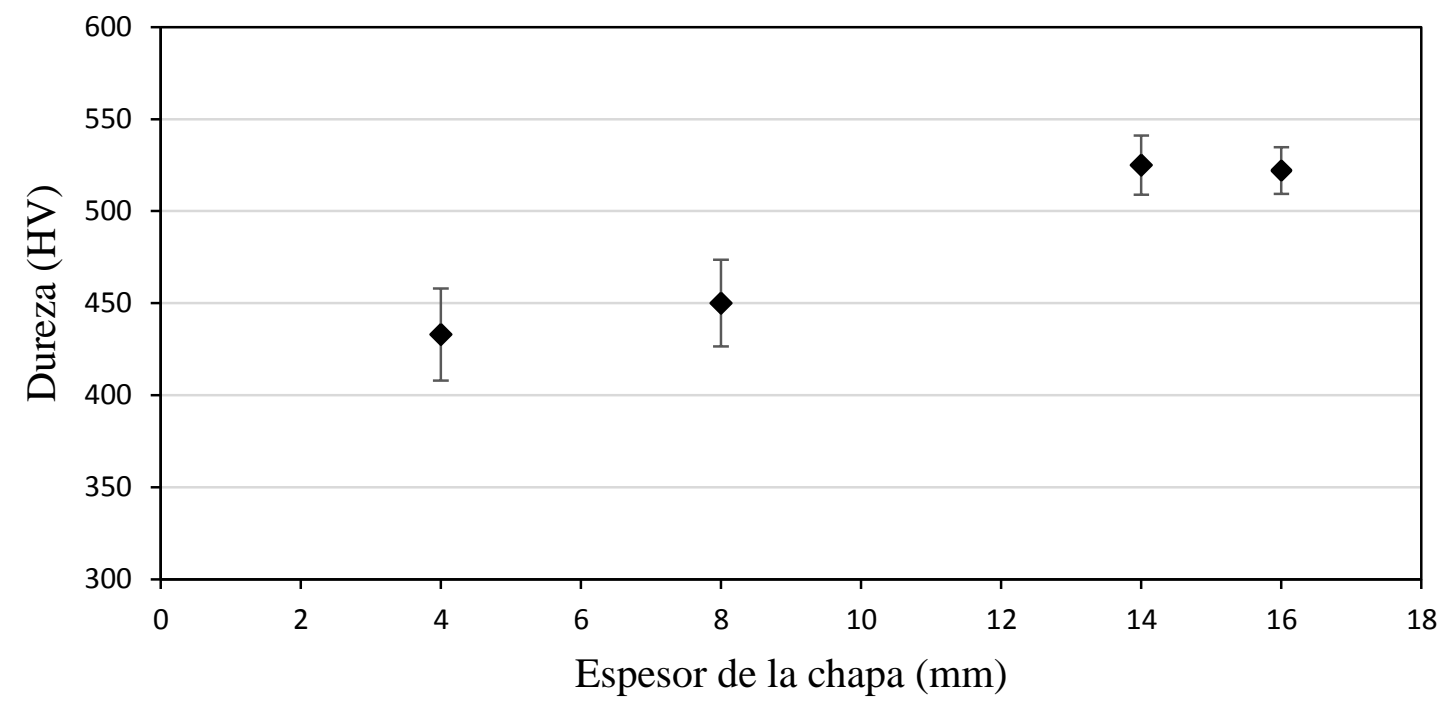

Figura 2. Comportamiento de la dureza media en la zona central de los depósitos de ensayo.

En el caso de los depósitos bajo estudio (Tabla 3), al ocurrir la cristalización de la austenita primaria se disuelve una cantidad limitada de carbono, cromo, manganeso y silicio; la mayor parte de estos elementos pasa hacia el líquido remanente en el cual cristaliza posteriormente la eutéctica constituida por carburos aleados y austenita. A medida que aumenta el subenfriamiento, debido a mayores velocidades de enfriamiento del depósito, disminuye la difusión, lo que implica un aumento en la heterogeneidad de los cristales formados, provocando diferencias en la estructura final de las aleaciones de los depósitos y por tanto en su dureza (Guliaev, 1978).

Por otra parte, el proceso de cristalización en estado sólido de la aleación, que ocurre cuando el metal atraviesa las temperaturas entre 1147 y $727{ }^{\circ} \mathrm{C}$, es también influenciado por la velocidad de enfriamiento del material del depósito. Durante el tiempo en el que la aleación transita por este rango, ocurre la segregación de las fases secundarias a partir del constituyente primario, las que se forman por la difusión de los elementos a partir de este (Guliaev, 1978). Al aumentar la velocidad de enfriamiento la segregación de fases que hay en exceso, comenzará a una temperatura cada vez más baja y como resultado los cristales segregados serán más pequeños y su cantidad menor, lo cual tiene un efecto en la composición fásica y, por tanto, en las propiedades mecánicas del material. Todo el análisis anterior permite fundamentar la variación resultante de la microestructura y la dureza en los depósitos obtenidos con diferentes condiciones de disipación de calor, establecidas por el espesor de la chapa donde se realizaron los ensayos. 
Es importante destacar que todas las características del depósito estudiadas (dilución, microestructura, dureza), tuvieron resultados similares en las muestras de los depósitos realizados sobre la chapa de espesor crítico $(14 \mathrm{~mm})$ y en la de espesor superior $(16 \mathrm{~mm})$, lo cual demuestra lo adecuado del empleo de los esquemas ideales de cuerpo y fuente de calor para la obtención del espesor a partir del cual la transferencia de calor desde el depósito de soldadura tiene un carácter tridimensional. Esto permite dimensionar la muestra de ensayo en función de la aplicación práctica del procedimiento de recargue, de manera que se simulen las condiciones de conducción del calor de los depósitos en la práctica, y con ello, la reproducibilidad del estudio de caracterización del material.

\section{Conclusiones}

En el dimensionamiento de la chapa para la realización de los depósitos de ensayo utilizados en la caracterización de depósitos de recargue comprendidos en el sistema de aleación de las fundiciones blancas, debe considerarse la influencia de las condiciones de disipación del calor, establecidas por el espesor del metal base, dado su efecto significativo en la composición química y microestructura del depósito, lo que se refleja en sus propiedades mecánicas.

El empleo de los esquemas simplificados de cuerpos y fuentes de calor en la soldadura, utilizados en la teoría clásica de los procesos térmicos para la descripción del fenómeno de transferencia de calor en la soldadura, permite determinar de manera satisfactoria el rango de espesor de la chapa de metal base en que se obtienen las condiciones de disipación de calor máxima y mínima, y con ello el empleo adecuado de las condiciones de ensayo para garantizar la reproducibilidad del estudio de caracterización del depósito en la práctica del recargue de piezas.

\section{Referencias Bibliográficas}

ASTM E3-11. (2011). Standard guide for preparation of metallographic specimens. Pennsylvania: American Society or Testing of Materials (ASTM).

ASTM E 562. (2011). Standard test method for determining volume fraction by systematic manual point count. West Conshohocken: American Society of Testing of Materials (ASTM).

AWS A5.13/A5.13M. (2010). Specification for surfacing electrodes for shielded metal arc welding. Miami: American Welding Society (AWS).

Collazo-Carceller, R., López-Salinas, H. (2009). Estudio del comportamiento de los recargues multicapas de depósitos soldados de fundición blanca al cromo. Revista Ingeniería Mecánica, 12(2), 83-92.

Chotěborský, R., Hrabě, P., Kabutey A. (2011). The effect of microstructure of the hypoeutectic Fe-Cr-C hardfacing on abrasive wear. Scientia Agric Bohemica, 42(3), 127-132.

Chotěborský, R., Hrabě, P., Müller, M., Savková, J., Jirka, M. (2008). Abrasive wear of high chromium Fe-Cr-C hardfacing alloys. Scientia Agriculturae Bohemica, 54(4), 192-198.

Dieter, G. E. (1997). Materials selection and design. ASM HandBook. Volume 20. 1st edition. EUA: ASM International.

Dulón-Gómez, J. (2003). Manual de soldadura pata la industria azucarera. La Habana: Ed. Talleres de Impresiones gráficas del Ministerio del Azúcar.

EN 1993-1-2. (2005). Eurocode 3: Design of steel structures - Part 1-2: general rules - structural fire design. Bruselas: European Comittee for Standardization (CEN).

Grong, O. (1997). Metallurgical modelling of welding. $2^{\text {nd }}$ edition. London: The Institute of Materials.

Gucwa, M., Winczek, J., Parzych, S., Kukuryk, M. (2020). The effect of the hardfacing processes parameters on the carbide volume fraction. Springer Nature Switzerland AG. 410-417.

Guliaev, A. (1978). Metalografía. Tomo I. Moscú: Editorial Mir.

Kou, S. (2002). Welding metallurgy. $2^{\text {nd }}$ edition. New Jersey: A John Wiley \& Sons, Inc. 
Marques, P. V., Modenesi, P. J. (2014). Algumas equações úteis em soldagem. Soldagem \& Inspeção. 19(01), 091-102.

Masubuchi, K. (1980). Analysis of welded structures. Oxford: Pergamon Press Ltd.

Matsuo, T. T., Kiminami, C. S., Botta-Fo, W. J., Bolfarini, C. (2005). Sliding wear of spray-formed highchromium white cast iron alloys. Wear, 259, 445-452.

Morsy, M., El-Kashif, E. (2014). The effect of microstructure on high-stress abrasion resistance of $\mathrm{Fe}-\mathrm{Cr}-\mathrm{C}$ hardfacing deposits. Weld World, 58, 491-497.

Oates, W. R., Saitta, A. M. (1998). Welding handbook AWS. Materials and application. Volume 4, part 2. $8^{\text {th }}$ edition. Miami: Ed. AWS.

Olson, L., Siewert, T. A., Liu, S., Edwards, G. (1993). Welding fundamentals and processes ASM handbook. Volume 06. EUA: ASM International.

Ortiz-Méndez, T. (2018). Desarrollo de un electrodo tubular revestido a partir de una ferroaleación del sistema $\mathrm{Fe}-\mathrm{Cr}$-Mn-Si-C para el recargue de piezas sometidas a desgaste abrasivo. Tesis doctoral. Santa Clara: Universidad Central "Marta Abreu" de las Villas.

Ortiz-Méndez, T., Cruz-Crespo, A., Rodríguez, M. (2019). Efecto del número de pasadas sobre el desempeño al desgaste micro-abrasivo de depósitos de recargue obtenidos con un electrodo tubular revestido experimental. Rev. Téc. Ing. Univ. Zulia, 42(1), 19-26.

Pozo-Morejón, J. A., Guimaraes, L. F. (2018). Ajuste de los calores de entrada que se corresponden con los tiempos de enfriamiento de la ZAT en soldadura GMAW sobre acero Dúplex 2205 empleando MEF. Soldagem \& Inspeção, 23(3), 413-422.

Radzikowska, J. M. (2004). ASM handbooks: metallography and microstructures. $3^{\text {th }}$ edition. Ohio: ASM International.

Raynor, G. V., Rivlin, V. G. (1988). Phase equilibria in iron ternary alloys: a critical assessment of the experimental literature. $1^{\text {st }}$ edition. London: Institute of Metals North American Publications Center.

Schön, C. G., Sinatora, A. (1998). Simulation of solidification paths in high chromium white cast irons for wear applications. Calphad, 22(4), 437-448.

Tabrett, C. P., Sare, I. R., Ghomashchi, M. R. (1996). Microstructure-property relationships in high chromium white iron alloys. International Materials Reviews, 4(2), 59-81.

Thum, E. E. (1935). Book of stainless steel. EUA: American Society of Materials.

Vedia, L. A., Svoboda, H. G. (2004). Introducción a la metalurgia de la soldadura. Monografía. Buenos Aires: Ed. Departamento de Mecánica.

Yüksel, N., Sahin, S. (2014). Wear behavior-hardness-microstructure relation of $\mathrm{Fe}-\mathrm{Cr}-\mathrm{C}$ and $\mathrm{Fe}-\mathrm{Cr}-\mathrm{C}-\mathrm{B}$ based hardfacing alloys. Materials and Design, 58, 491-498. 


\section{REVISTA TECNICA}

DE LA FACULTAD DE INGENIERIA

UNIVERSIDAD DEL ZULIA

Vol. 44. $\mathbf{N}^{\circ} 3$, Septiembre - Diciembre, 2021

Esta revista fue editada en formato digital y publicada en Agosto 2021, por el Fondo Editorial Serbiluz, Universidad del Zulia. Maracaibo-Venezuela

www.luz.edu.ve

www.serbi.luz.edu.ve

www.produccioncientificaluz.org 